TRANSACTIONS OF THE

AMERICAN MATHEMATICAL SOCIETY

Volume 350, Number 9, September 1998, Pages 3537-3553

S 0002-9947(98)02274-0

\title{
POINCARÉ EMBEDDING OF THE DIAGONAL
}

\author{
YANGHYUN BYUN
}

\begin{abstract}
There is a Poincaré embedding structure on the diagonal $X \rightarrow$ $X \times X$ under the conditions: i) $X$ is formed by gluing two compact smooth manifolds along their boundaries using a homotopy equivalence and ii) a square-root closed condition is satisfied by the fundamental groupoid of the boundary.
\end{abstract}

\section{INTRODUCTION}

Let $M$ be a smooth manifold and $\Delta: M \rightarrow M \times M$, the diagonal embedding. Then there is a tubular neighborhood $U$ of $\Delta M$, with a retraction $r: U \rightarrow \Delta M=$ $M$, so that the tangent sphere bundle of $M$ is isomorphic to the one whose projection is $\left.r\right|_{\partial U}$. This provides a Poincaré embedding structure, as described in 1.1 below, on the diagonal, which suggests that, for a Poincaré complex in general, a Poincaré embedding structure on the diagonal might give rise to something like a tangent fibration.

In this paper, a Poincaré pair is in the sense of C.T.C. Wall ([12]). A CWcomplex $X$ is referred to as a Poincaré complex if $(X, \emptyset)$ is a Poincaré pair. For any sphere fibration $\xi$ over a topological space, $S \xi$ denotes the total space and $D \xi$, the mapping cylinder of the projection. The following definition is essentially due to Wall (p.113, [13]):

Definition 1.1. Given Poincaré pairs, $(A, B),(X, Y)$, respectively of dimension $n$ and $n+q$, and a continuous map $f:(A, B) \rightarrow(X, Y)$, a Poincaré embedding structure on $f$ consists of:

a $(q-1)$-sphere fibration $\nu$ with projection $p: S \nu \rightarrow A$,

a Poincaré pair $\left(X^{\prime}, S \nu \cup_{\left.S \nu\right|_{B}} Y^{\prime}\right)$ of dimension $n+q$,

a homotopy equivalence $h:\left(D \nu \cup_{S \nu} X^{\prime},\left.D \nu\right|_{B} \cup_{S_{\left.\nu\right|_{B}}} Y^{\prime}\right) \rightarrow(X, Y)$, so that the following diagram commutes up to homotopy:

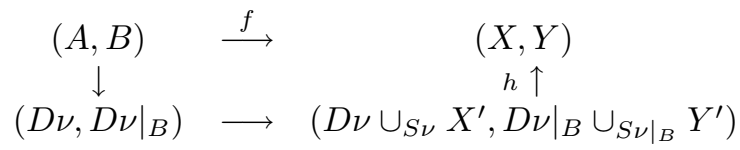

where the unmarked arrows are the inclusions.

In particular, we shall call $\nu$ and $\left(X^{\prime}, S \nu \cup_{\left.S \nu\right|_{B}} Y^{\prime}\right)$, respectively, the normal fibration and the complement of the Poincaré embedding structure on $f$.

One might be tempted to define the tangent fibration of a Poincaré complex $X$ as the normal fibration of a Poincaré embedding structure on the diagonal $\Delta: X \rightarrow$

Received by the editors July 29, 1996.

1991 Mathematics Subject Classification. Primary 57P10.

Partially supported by 1996 Faculty Research Fund of Hanyang University, Korea.

(C)1998 American Mathematical Society 
$X \times X$. However, it is not yet known whether the diagonal always admits a Poincaré embedding structure. Even if Poincaré embedding structures on $\Delta$ do exist, it is not clear whether the normal fibrations of two Poincaré embedding structures are necessarily fiber homotopy equivalent to each other, since the sphere fibrations are not in the stable range (see, for example, p.115, [13]). In fact, the author has shown that this is indeed so, which consists of his $\mathrm{Ph} . \mathrm{D}$. thesis together with this paper. (The nature of the argument, however, is significantly different from that of the current paper; the former is more algebraic than geometric while the latter is exactly the opposite. Therefore, it seems appropriate to regard each of the two as an independent paper.)

This paper will concentrate on the problem of whether the diagonal $\Delta: X \rightarrow$ $X \times X$ admits a Poincaré embedding structure.

In fact, there are results such as [5], [7], [8] which assert that: given any continuous map $f: Y \rightarrow X$ between finite Poincaré complexes of formal dimension $i$ and $n$, respectively, with $n \geq 2 i+1$, there exists a Poincaré embedding structure on $f$, in particular, if $X$ is simply connected. Unfortunately they do not apply to our situation, because of their assumptions on the fundamental group and on the dimension.

We will consider the case when the Poincaré complex $X$ under study is formed by gluing two smooth manifolds along their boundaries using a homotopy equivalence (see 4.1). Also we shall demand an extra condition:

Definition 1.2. Let $H<G$ be groupoids. We will say $H$ satisfies the square-root closed condition in $G$ if ' $a{ }^{2} \in H$ ' implies ' $a \in H$ ' for any $a \in G$. In particular, we will say a groupoid $G$ satisfies the diagonal square-root closed condition if ' $a{ }^{2}=b^{2}$, implies ' $a=b$ ' for any $a, b \in G$, that is, if the diagonal subgroupoid $\Delta G<G \times G$ satisfies the square-root closed condition.

The square-root closed condition above played an important role in the study of the splitting problem by Cappell ([2]).

The following is the main theorem of the paper.

Theorem 1.3. Let $(X, Y)$ be a Poincaré pair, $\operatorname{dim} X \geq 4$, such that $(X, Y)=$ $\left(M_{0} \cup_{f} M_{1}, \partial^{-} M_{0} \sqcup \partial^{+} M_{1}\right)$, where $\left(M_{i} ; \partial^{-} M_{i}, \partial^{+} M_{i} ; \emptyset\right), i=0,1$, are manifold triads and $f: \partial^{+} M_{0} \rightarrow \partial^{-} M_{1}$ is a homotopy equivalence. Assume the fundamental groupoid of $\partial^{+} M_{0}$ satisfies the diagonal square-root closed condition. Then there exists a Poincaré embedding structure on the diagonal $\Delta:(X, Y) \rightarrow(X, Y) \times$ $(X, Y)$.

Here the square-root closed condition is needed not because of the Cappell's splitting theorem (see 5.1 below) but because of an embedding problem:

Lemma 1.4. Assume that $M^{n}, K^{2 n-1} \subset N^{2 n}, n \geq 3$, are connected compact smooth manifolds and that $\partial K=K \cap \partial N, K$ is transversal to $\partial N$ and the normal bundle $K$ in $N$ is trivial. Let $f: M \rightarrow N$ be a continuous map such that $\left.f\right|_{\partial M}$ is an embedding into $\partial K \subset N$ and $f$ factors through $f^{\prime}: M \rightarrow K$ by the inclusion $K \hookrightarrow N$ up to homotopy rel $\partial M$. Then, if $f_{*}^{\prime} \pi_{1}(M)<\pi_{1}(K)$ satisfies the squareroot closed condition, $f$ is homotopic to an embedding rel $\partial M$.

As an immediate consequence, we have: 
Corollary 1.5. Let $M^{n}, N^{2 n}, n \geq 3$, be connected compact smooth manifolds and $F: M \times I \rightarrow N$ be a homotopy such that $F_{0}$ and $F_{1}$ are embeddings. Assume

$$
\left(F_{0}\right)_{*} \pi_{1}(M)<\pi_{1}(N) \text { satisfies the square-root closed condition. }
$$

Then, the map $\bar{F}: M \times I \rightarrow N \times[-1,1] \times I$, defined by $\bar{F}(x, t)=(F(x, t), 0, t)$, is homotopic to a concordance rel $M \times\{0,1\}$.

Here a concordance means simply an embedding.

At the moment, it is far from being clear how essential the square-root closed condition is in 1.3 above. For example, one may alter the manifolds $M_{0}, M_{1}$ and $\pi_{1}\left(\partial^{+} M_{0}\right)=\pi_{1}\left(\partial^{-} M_{1}\right)$ by trading handles of index at most two along $\partial^{+} M_{0}$ (or $\partial^{-} M_{1}$ ) preserving the homotopy type of the Poincaré pair: One may assume $f$ sends a regular neighborhood of any 1-complex in $\partial^{+} M_{0}$ to such in $\partial^{-} M_{1}$ by applying a splitting theorem, ${ }^{1}$ and $f$ can be assumed as a diffeomorphism when restricted to such a regular neighborhood since the fundamental group of $G / O$ is trivial. ${ }^{2}$

Nevertheless this does not mean that one may alter $\partial^{+} M_{0}$ so that its fundamental group satisfies the diagonal square-root closed condition. The fact that such as the square-root closed condition is needed in 1.3 makes it seem more plausible that: there might be a finite Poincaré complex for which the diagonal does not admit any Poincaré embedding.

The author would like to thank his advisor, Professor Frank Connolly at Notre Dame, for his help in writing this paper. Among other things, his suggestions have brought a substantial improvement to the style of the paper. The author is more than grateful also for the encouragement and the inspiring conversations Professor Connolly has provided throughout his graduate years.

\section{GLuing SPACES}

We start by introducing the notion of gluing:

Definition 2.1. Let $(X, A)$ be a pair and $Y$, a space. If $f: A \rightarrow Y$ is a continuous map, we define $X \cup_{f} Y$ as the quotient space of the disjoint union $X \sqcup Y$ with respect to the equivalence relation generated by $a \sim f(a)$. We will often refer to $X \cup_{f} Y$ as the space formed by gluing $X$ to $Y$ using $f$.

The following is well-known (for example, see [9]) and we omit the proof.

Lemma 2.2. Let $(X, A),(Y, B)$ be NDR-pairs and $V, W$, spaces. Assume the following diagram commutes up to homotopy:

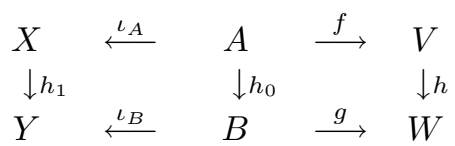

in which vertical arrows are homotopy equivalences and $\iota_{A}, \iota_{B}$ are the inclusions. Then $X \cup_{f} V$ and $Y \cup_{g} W$ are homotopy equivalent to each other.

The following is also well known (for example, see 2.5, 6, [4], also 2.1, [12]):

\footnotetext{
${ }^{1}$ Wall's result will be enough if we assume $f$ is a simple homotopy equivalence and in general one may use Cappell's result.

${ }^{2}$ The author would like to thank the anonymous referee for his kind explanation of this technique of altering $\pi_{1}\left(\partial^{+} M_{0}\right)$.
} 
Lemma 2.3. Let $\left(X_{i}, A_{i}^{-} \cup A_{i}^{+}\right), i=0,1$, be n-dimensional Poincaré pairs. Furthermore, writing $B_{i}=A_{i}^{-} \cap A_{i}^{+}$, assume $\left(A_{i}^{ \pm}, B_{i}\right)$ are Poincaré pairs. If $f$ : $\left(A_{0}^{+}, B_{0}\right) \rightarrow\left(A_{1}^{-}, B_{1}\right)$ is a homotopy equivalence, then $\left(X_{0} \cup_{f} X_{1}, q\left(A_{0}^{-} \sqcup A_{1}^{+}\right)\right)$is an n-dimensional Poincaré pair in which $q$ is the quotient map.

Let $A, A^{\prime}$ be respectively subspaces of $X$ and $X^{\prime}$. We will say $f: X \rightarrow X^{\prime}$ is a homotopy equivalence rel $A$ if $(a) f$ restricts to a homeomorphism $\left.f\right|_{A}: A \rightarrow A^{\prime},(b)$ there is a homotopy inverse $g: X^{\prime} \rightarrow X$ such that $\left.g\right|_{A^{\prime}}: A^{\prime} \rightarrow A$ is the inverse of $\left.f\right|_{A}$, and, furthermore, $(c)$ there are homotopies $H$ from $g f$ to $1_{X}$ and $H^{\prime}$ from $f g$ to $1_{X^{\prime}}$ such that $H(\cdot, t)$ and $H^{\prime}(\cdot, t)$ are the identity maps on $A$ and $A^{\prime}$ respectively for any $t \in I$.

Let $X, A$ be spaces such that $A \subset X$. We will call a closed neighborhood $N$ of $A$ in $X$ a collar of $A$ if $(N, A)$ and $(A \times I, A \times\{0\})$ are homeomorphic to each other as pairs.

The following will be useful for the later discussions, in particular, because of its 'relative' clause. We omit the proof.

Proposition 2.4. Let $(X, A)$ be a pair and $Y$, a space. Assume $A$ has a collar in $X$ identified with $A \times I$, where $A$ is identified with $A \times\{0\}$. Assume $f, g$ are continuous maps from $A$ to $Y$ homotopic to each other. Then, $X \cup_{f} Y$ and $X \cup_{g} Y$ are homotopy equivalent to each other $\operatorname{rel}(X-A \times[0,1)) \sqcup Y$.

The following and the subsequent remark will be needed for the proof of Claim 4.A below.

Lemma 2.5. Let $X=A \cup(B \times I) \cup C$ be a space for which $A \cap(B \times I)=B \times\{1\}$ and $(A \cup(B \times I)) \cap C=B \times\{0\}$. Let $Y$ be another space. Assume $f, g: X \rightarrow Y$ are continuous maps homotopic to each other rel $A \cup(B \times I)$. Regard $f, g$ as maps from $X \times\{0\}$ up to the identification $X \times\{0\}=X$. Then $(X \times I) \cup_{f} Y$ is homotopy equivalent to $(X \times I) \cup_{g} Y$ rel $X \times\{1\} \cup q((A \times I) \sqcup Y)$, where $q$ denotes the quotient map.

We only sketch the proof of this lemma by providing:

$A$ construction of a homotopy equivalence $\alpha:(X \times I) \cup_{f} Y \rightarrow(X \times I) \cup_{g} Y$ : Assume a homotopy $H: X \times I \rightarrow Y$ is given so that $H_{0}=f, H_{1}=g, H(x, t) \equiv$ $f(x, t)=g(x, t)$, for all $x \in A \cup(B \times I), t \in I$.

Define a continuous map $h: I \times I \rightarrow I \times I$ as follows: $h(s, t)=(s, 0)$ if $t \leq \frac{1}{2}-\frac{s}{2}$ and $h(s, t)=\left(s, \frac{2 t+s-1}{1+s}\right)$ if $t \geq \frac{1}{2}-\frac{s}{2}$. Then $h$ is well-defined. Also note that $h$ restricted to $(I \times\{0\}) \cup(\{1\} \times I) \cup(I \times\{1\})$ is the identity.

Define $\alpha:(X \times I) \cup_{f} Y \rightarrow(X \times I) \cup_{g} Y$ as follows: $\alpha[y]=[y]$ for any $y \in Y$, $\alpha[c, t]=[H(c, 2 t)]$ for any $(c, t) \in C \times\left[0, \frac{1}{2}\right], \alpha[c, t]=[c, 2 t-1]$ for any $(c, t) \in$ $C \times\left[\frac{1}{2}, 1\right], \alpha[b, s, t]=[b, h(s, t)]$ for any $(b, s, t) \in(B \times I) \times I$, and finally $\alpha[a, t]=[a, t]$ for any $(a, t) \in A \times I$.

Then $\alpha$ is well-defined. In particular, for any $(b, 0) \in(B \times I) \cap C$, we have that

$$
\begin{gathered}
{[b, h(0, t)]=[b, 0,0]=[f(b, 0)]=[g(b, 0)]=[H((b, 0), 2 t)] \text { if } t \in\left[0, \frac{1}{2}\right],} \\
{[b, h(0, t)]=[b, 0,2 t-1]=[(b, 0), 2 t-1] \text { if } t \in\left[\frac{1}{2}, 1\right] .}
\end{gathered}
$$

Also note that $\alpha$ restricted to $q(X \times\{1\} \sqcup(A \times I) \sqcup Y) \subset(X \times I) \cup_{f} Y$ is the homeomorphism induced by the identity. 
Then the proof of 2.5 can be completed by defining (i) an inverse $\hat{\alpha}:(X \times I) \cup_{g}$ $Y \rightarrow(X \times I) \cup_{f} Y$ in the same manner as $\alpha$, replacing $H$ with $\bar{H}$, which is defined by $\bar{H}(x, t)=H(x, 1-t)$, and using the same $h$; and (ii) the homotopies between $\hat{\alpha} \alpha, \alpha \hat{\alpha}$ and the identities which respectively fix the images of $X \times\{1\} \sqcup(A \times I) \sqcup Y$ by the quotient maps.

Remark 1. In particular, we will use the fact that, in the construction above, we have:

$$
\begin{aligned}
& \alpha[c, t]=[H(c, 2 t)] \text { for any }(c, t) \in C \times\left[0, \frac{1}{2}\right], \\
& \alpha[c, t]=[c, 2 t-1] \text { for any }(c, t) \in C \times\left[\frac{1}{2}, 1\right] .
\end{aligned}
$$

\section{Proof of 1.4}

First of all, we need to recall a result by Haefliger (4.1 Théorème, [3]):

Theorem 3.1. [A. Haefliger] Assume $V, M$ are smooth manifolds of dimension $n$, $m$, respectively, and assume $V$ is compact. Suppose $2 m \geq 3(n+1)$. Let $f: V \rightarrow M$ be a continuous map such that $f$ is an embedding in a neighborhood of $\partial V$ and $f(\partial V) \cap f(V-\partial V)=\emptyset$. Assume $\pi_{i}(f)=0$ for $0 \leq i \leq 2 n-m+1$. Then $f$ is homotopic to an embedding relative to a neighborhood of $\partial V$.

Proof of 1.4. Since $n \geq 3$, by a result of Whitney ([15]), up to homotopy rel $\partial M$, we may assume $f^{\prime}: M \rightarrow K$ is a smooth map such that

i) $f^{\prime}$ is self-transverse,

ii) $f^{\prime}$ has no triple point, and

iii) $f^{\prime}$ is an embedding at the complement of the union of some finite disjoint circles embedded in $M$.

To be more precise, according to [15], there are three ways in which $f^{\prime}$ may fail to be an embedding at those circles:

Type $A: f^{\prime}$ restricts to $\left.f^{\prime}\right|_{C}: C \rightarrow l$, where $C$ is an embedded circle in $M$ and $l$ is a line segment embedded in $N^{\prime}$ so that each interior point of $l$ is a double point and $d f_{x}^{\prime}: T_{x} M \rightarrow T_{f^{\prime}(x)} N$ is injective for all $x \in f^{\prime-1}$ Int $l$ and $d f_{x}^{\prime}\left(T_{x} C\right)=0$ if $f^{\prime}(x) \in \partial l$.

Type $B: f^{\prime}$ restricts to $\left.f^{\prime}\right|_{\tilde{C}}: \tilde{C} \rightarrow C$, which is a double covering.

Type $C: f^{\prime}$ restricts to $\left.f^{\prime}\right|_{C \sqcup C^{\prime}}: C \sqcup C^{\prime} \rightarrow \bar{C}$, a double covering, where $C$ and $C^{\prime}$ are embedded disjoint circles in $M$ and $\bar{C}$ is an embedded circle in $N$.

We start by arranging that $f^{-1} \partial K=\partial M$, which can be done using some collar neighborhoods of $\partial M$ and $\partial K$. Then, step by step, we will replace $f$, up to homotopy rel $\partial M$, with another which has fewer circles on which $f$ fails to embed:

Step 1 . Let $C, l$ be as in Type $A$. Take a regular neighborhood $U \subset N$ of $l$ so that $U$ is disjoint from those other circles or the other line segments in $N$ as described above, and also from $\partial N$.

Note that $U$ is diffeomorphic to $D^{2 n}$ and, in particular, simply connected.

Let $V_{1}$ be the path component of $f^{-1} U$ containing $C$. And let $V_{i}, i=2,3, \cdots, k$, be the path components other than $V_{1}$. Then $f$ restricted to $\bigsqcup_{i=2}^{k} V_{i}$ is an embedding. Remove $f\left(V_{i}\right)$ from $U$ for each $i=2,3, \cdots, k$. Denote the resulting smooth neighborhood of $l$ by $U^{\prime}$. Note that the fundamental group of $U^{\prime}$ is the same as $U^{\prime}$ 's, since $\operatorname{dim} U-\operatorname{dim} V_{i} \geq 3$. Then the restriction of $f$, say, $f_{U^{\prime}}: V_{1} \rightarrow U^{\prime}$, is a continuous map which is an embedding on a neighborhood of $\partial V_{1}$. Since $U^{\prime}$ is 


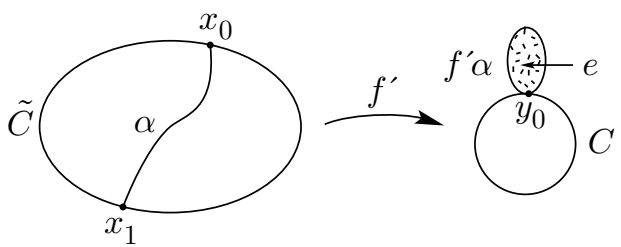

Figure 1

simply connected, $f_{U^{\prime}}$ is homotopic to an embedding, relative to a neighborhood of $\partial V_{1}$, by 3.1 .

Thus we may assume $f$ has one fewer circle of Type A.

Note that, even after performing this processes, all of those line segments or circles in $N$ as described in Type A, B, C above lie in $K \subset N$. Furthermore, we can still find a regular neighborhood in $N$ of a line segment, if any remain, of Type A which is disjoint from other lines or circles as described in Types A, B, C and, in particular, disjoint from the closure of $f M-K$ in $N$ and also from $\partial N$

Therefore, we may repeatedly apply the above process to obtain a new $f$ which does not have any circle of Type A.

Step 2. Now let $\tilde{C} \subset M, C \subset K$ as in Type B. Choose $y_{0} \in C$ and write $f^{-1} y_{0}=$ $\left\{x_{0}, x_{1}\right\} \subset M$. Then, first of all, we observe:

Claim. We may choose a path $\alpha:[0,1] \rightarrow M, \alpha(0)=x_{0}, \alpha(1)=x_{1}$, so that $\left[f^{\prime} \alpha\right] \in \pi_{1}\left(K, y_{0}\right)$ is the identity.

Proof. Let $\gamma$ be the path from $x_{1}$ to $x_{0}$ along one of the semicircles of $\tilde{C}$. Then $f^{\prime} \gamma$ is a loop in $K$ based on $y_{0}$.

In particular, $\left[f^{\prime} \gamma\right] \in f_{*}^{\prime} \pi_{1}(M)<\pi_{1}(K)$ since $f_{*}^{\prime} \pi_{1}(M)<\pi_{1}(K)$ satisfies the square-root closed condition. Therefore, we choose $\alpha$ as a path from $x_{0}$ to $x_{1}$ so that $[\gamma * \alpha] \in \pi_{1}\left(M, x_{0}\right)$ is such that $f_{*}^{\prime}[\gamma * \alpha] \in \pi_{1}\left(K, y_{0}\right)$ is $\left[f^{\prime} \gamma\right]$ itself.

We may assume $\alpha:[0,1] \rightarrow M$ is smoothly embedded and that $\operatorname{Im} \alpha$ is disjoint from circles of Types B, C other than $\tilde{C}$. Furthermore, arrange so that $\left.\alpha_{*} \frac{d}{d t}\right|_{t=i} \notin$ $T_{x_{i}} \tilde{C}$, for $i=0,1$.

Note that, even if we may not assume $f(M) \subset K$ any longer, still we may choose a smooth embedding $e: D^{2} \rightarrow K$, and furthermore we may choose it so that

$$
\operatorname{Im} e \cap \operatorname{Im} f=\operatorname{Im} f \alpha \text { and } e_{*} T_{x_{i}} D^{2} \cap T_{y_{0}} C=\{0\}, \quad i=0,1 .
$$

See Figure 1.

Then we construct a regular neighborhood $U$ of $C \cup D^{2}$ in $N$ so that it is disjoint from other circles or lines in $N$ as described in Types B, C and also disjoint from the closure of $f M-K$ in $N$ and from $\partial N$.

As before, let $V_{1}$ be the path component of $f^{-1} U$ containing $C$ and let $V_{i}$, $i=2,3, \cdots, k$, be the path components other than $V_{1}$. Remove $f V_{i}$ 's, $i>1$, from $U$ and call the resulting space $U^{\prime}$. Then clearly the restriction of $f$, say, $f_{U^{\prime}}: V_{1} \rightarrow U^{\prime}$, induces a surjection between the fundamental groups. Therefore, we may apply 3.1 .

As in Step 1, we may repeat this process to remove all the circles of Type $B$.

Step 3. Assume $C, C^{\prime}, \bar{C}$ are as in Type C. Choose a Riemannian metric on $M$ and take disjoint normal neighborhoods $U$ and $U^{\prime}$ of $C$ and $C^{\prime}$, respectively, whose 
points are identified with the normal vectors to $C, C^{\prime} \subset M$ with length $\leq \delta$ for some $\delta>0$ by the exponential map. By choosing $\delta$ small enough we may assume $f U, f U^{\prime}$ are disjoint from other circles of Type $\mathrm{C}$ and also from the closure of $f M-K$ in $N$.

Now identify a neighborhood of $K$ with $K \times[-1,1]$ using a diffeomorphism, which is possible by the assumption. Then there is a positive number $\epsilon$ so that both $f U \times[-\epsilon, \epsilon], f U^{\prime} \times[-\epsilon, \epsilon] \subset K \times[-1,1]$ are disjoint from the closure of $f M-K$ in $N$.

Let $\varphi:[0, \delta] \rightarrow[0, \epsilon]$ be a decreasing smooth function whose value is constantly $\epsilon$ in a neighborhood of 0 and constantly 0 in a neighborhood of $\delta$. Then we define $g: M \rightarrow N$ by the rule:

$$
\begin{gathered}
g(v)=(f(v), \varphi(|v|)) \text { for any } v \in U, \\
g(v)=(f(v),-\varphi(|v|)) \text { for any } v \in U^{\prime},
\end{gathered}
$$

and $g \equiv f$ elsewhere. Clearly $g$ is homotopic to $f$. We take this $g$ as our new $f$.

We repeat this process until we exhaust all the circles of Type $\mathrm{C}$ to make $f$ an embedding.

\section{Constructions}

In this section, we prove 1.3. Also other results regarding the existence of Poincaré embedding structures (in particular, 4.8,9) are obtained. The main tools are provided by a splitting theorem by S.E. Cappell ( 5.1 below), an embedding theorem by A. Haefliger (3.1 above), and 1.5 above.

Definition 4.1. Let $(X, \partial X)$ be an $n$-dimensional Poincaré pair. We will say $(X, \partial X)$ admits a decomposition by two smooth manifolds if

$i)$ there are smooth manifold triads $\left(P ; P_{0}, P_{1} ; \partial P_{0}\right),\left(Q ; Q_{0}, Q_{1} ; \partial Q_{0}\right)$ together with a homotopy equivalence $\varphi:\left(P_{0}, \partial P_{0}\right) \rightarrow\left(Q_{0}, \partial Q_{0}\right)$ so that

ii) $(X, \partial X)$ is homotopy equivalent to $\left(P \cup_{\varphi} Q, q\left(P_{1} \sqcup Q_{1}\right)\right)$, where $q$ is the quotient map (note that $\partial P_{0}=\partial P_{1}$ and $\partial Q_{0}=\partial Q_{1}$ ).

In the above, $P, Q$ are allowed to have more than one path component. By 2.3, any pair which admits a decomposition by two smooth manifolds is a Poincaré pair. In fact, it is a patch space as defined by L. Jones ([6]).

We will make use of a more general form of the idea 'regular neighborhood' and a slightly altered notion of the usual splitting ([2] or [13]):

Definition 4.2. $i$ ) Let $B \subset N$ be compact smooth manifolds such that $B \subset \operatorname{Int} N$. $U_{B} \subset$ Int $N$ is an h-regular neighborhood of $B$ if $\left(U_{B}, \partial U_{B}\right)$ is a compact smooth neighborhood of $B$ such that $B$ is a strong deformation retract of $U_{B}$ and the inclusion $\partial U_{B} \hookrightarrow U_{B}$ has a spherical homotopy fiber.

ii) Let $(M, \partial M),(N, \partial N)$ be compact smooth manifolds and $f:(M, \partial M) \rightarrow$ $(N, \partial N)$, a homotopy equivalence. Also let $B \subset N$ be a submanifold which lies in Int $N$. We will say that $f$ is $h$-split along $B$ if there is an $h$-regular neighborhood $U_{B} \subset$ Int $N$ of $B$ so that, writing $f^{-1} U_{B} \equiv U_{A}, U_{A} \subset M$ is a compact smooth manifold and the restrictions of $f$ to

$\left(U_{A}, \partial U_{A}\right) \rightarrow\left(U_{B}, \partial U_{B}\right), \quad\left(M-\operatorname{Int} U_{A}, \partial M \sqcup \partial U_{A}\right) \rightarrow\left(N-\operatorname{Int} U_{B}, \partial N \sqcup \partial U_{B}\right)$ are homotopy equivalences. We shall refer to $U_{B}$ as the $h$-regular neighborhood of $B$ associated to the $h$-splitting of $f$. 


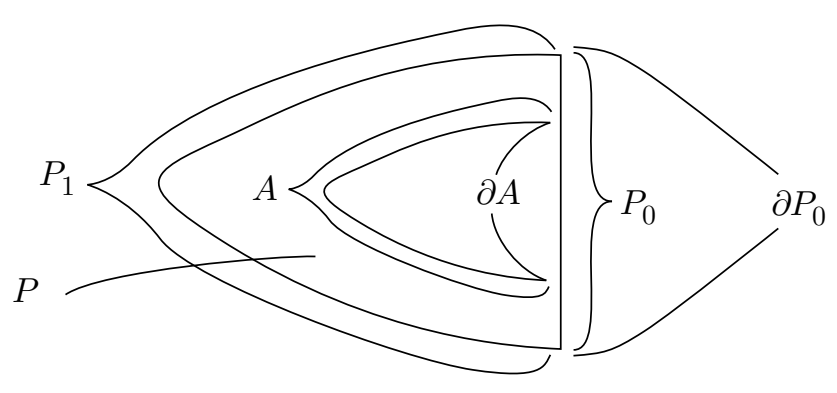

FIGURE 2

iii) If $f$ is homotopic rel $\partial M$ to a map which is $h$-split along $B$, then we will say $f$ is $h$-splittable along $B$.

Standing Hypotheses. (See Figure 2.) Throughout the following, from 4.3 to 4.9 ,

(a) $\left(P ; P_{0}, P_{1} ; \partial P_{0}\right),\left(Q ; Q_{0}, Q_{1} ; \partial Q_{0}\right)$ are fixed compact smooth manifold triads.

(b) $\varphi:\left(P_{0}, \partial P_{0}\right) \rightarrow\left(Q_{0}, \partial Q_{0}\right)$ is a fixed homotopy equivalence. Also,

(c) $(A, \partial A),(B, \partial B)$ are fixed submanifolds of $\left(P, P_{0}\right),\left(Q, Q_{0}\right)$ such that $A \subset$ $P-P_{1}, B \subset Q-Q_{1}$. (Note that $\partial A \subset$ Int $P_{0}$ and $\partial B \subset$ Int $Q_{0}$.)

$(d)$ Furthermore, we assume that $\varphi$ restricts to a homotopy equivalence $\varphi^{\prime}$ : $\partial A \rightarrow \partial B$.

We will write $(X, \partial X) \equiv\left(P \cup_{\varphi} Q, q\left(P_{1} \sqcup Q_{1}\right)\right)$ and $Y \equiv A \cup_{\varphi^{\prime}} B$, where $q$ is the quotient map. We will denote the canonical injection $Y \rightarrow(X, \partial X)$ by $\iota$.

Goal. We will try to construct a Poincaré embedding structure (see 1.1) on $\iota$ : $(Y, \emptyset) \rightarrow(X, \partial X)$.

Construction 4.3. As a first step, we consider the case in which

$$
\varphi:\left(P_{0}, \partial P_{0}\right) \rightarrow\left(Q_{0}, \partial Q_{0}\right)
$$

is $h$-split along $\partial B \subset Q_{0}$. Under this assumption, we exhibit a Poincaré embedding structure on $\iota: Y \rightarrow(X, \partial X)$ as follows.

Let $U_{\partial B}$ be an $h$-regular neighborhood of $\partial B$ associated to the $h$-splitting of $\varphi$, and write $U_{\partial A} \equiv \varphi^{-1} U_{\partial B}$. Extend them to $h$-regular neighborhoods $\left(U_{A}, U_{\partial A}\right) \subset$ $\left(P, P_{0}\right)$ and $\left(U_{B}, U_{\partial B}\right) \subset\left(Q, Q_{0}\right)$ respectively of $(A, \partial A)$ and $(B, \partial B)$ so that $U_{A} \cap$ $P_{0}=U_{\partial A}, U_{B} \cap Q_{0}=U_{\partial B}$ and $U_{A} \cap P_{1}=U_{B} \cap Q_{1}=\emptyset$.

Note that $\varphi$ restricts to the homotopy equivalences:

$$
\partial U_{\partial A} \rightarrow \partial U_{\partial B}, \quad U_{\partial A} \rightarrow U_{\partial B}, \quad\left(P_{0}-\text { Int } U_{\partial A}, \partial P_{0}\right) \rightarrow\left(Q_{0}-\text { Int } U_{\partial B}, \partial Q_{0}\right) .
$$

Write

$$
\begin{gathered}
\partial_{1} U_{A} \equiv \partial U_{A}-\operatorname{Int} U_{\partial A}, \quad \partial_{1} U_{B} \equiv \partial U_{B}-\operatorname{Int} U_{\partial B}, \\
\left(U_{Y}, \partial U_{Y}\right) \equiv\left(U_{A} \cup_{\left.\varphi\right|_{U_{\partial A}}} U_{B}, \partial_{1} U_{A} \cup_{\left.\varphi\right|_{\partial U_{\partial A}}} \partial_{1} U_{B}\right), \\
K \equiv\left(P-\operatorname{int} U_{A}\right) \cup_{\left.\varphi\right|_{P_{0}-\operatorname{Int} U_{\partial A}}}\left(Q-\operatorname{int} U_{B}\right),
\end{gathered}
$$

in which Int $U_{A}$ and Int $U_{B}$ respectively denote the set of interior points of $U_{A}$ and $U_{B}$ as subspaces of $P$ and $Q$.

Clearly, $U_{Y}, K \subset X$ define a decomposition of $(X, \partial X)$. In particular, note that $U_{Y} \cap K=\partial U_{Y}$. 
Both $\partial_{1} U_{A} \hookrightarrow U_{A}, \partial_{1} U_{B} \hookrightarrow U_{B}$ have the homotopy fiber $S^{k-1}, k=\operatorname{dim} P-$ $\operatorname{dim} A$. Furthermore, $\partial U_{\partial A} \hookrightarrow U_{\partial A}, \partial U_{\partial A} \hookrightarrow U_{\partial A}$ also have the homotopy fiber $S^{k-1}$ and $\varphi$ induces a homotopy equivalence $\left(U_{\partial A}, \partial U_{\partial A}\right) \rightarrow\left(U_{\partial B}, \partial U_{\partial B}\right)$. Therefore we conclude that $\partial U_{Y} \hookrightarrow U_{Y}$ has the homotopy fiber $S^{k-1}$ (see, for example, $[10])$.

Let $\xi$ be the sphere fibration obtained by restricting the base space to $Y \subset U_{Y}$ from the Serre fibration of the inclusion $\partial U_{Y} \hookrightarrow U_{Y}$. Then $\left(U_{Y}, \partial U_{Y}\right)$ is naturally homotopic to $(D \xi, S \xi)$ by a homotopy equivalence which is the identity map when restricted to $Y \rightarrow Y \subset D \xi$. By replacing $\left(U_{Y}, \partial U_{Y}\right)$ with $(D \xi, S \xi)$, we obtain a Poincaré embedding structure on $\iota: Y \rightarrow(X, \partial X)$.

There is an obvious equivalence relation between Poincaré embedding structures: Let $f: X \rightarrow Y$ be a continuous map between Poincaré complexes, and let two Poincaré embedding structures $D \nu \cup_{S \nu} W, D \nu^{\prime} \cup_{S \nu^{\prime}} W^{\prime}$ be given. Assume there is a homotopy equivalence $D \nu \cup_{S \nu} W \rightarrow D \nu^{\prime} \cup_{S \nu^{\prime}} W^{\prime}$ which respects the decomposition and restricts to the identity map $X \rightarrow X$, understanding $X \subset D \nu, X \subset D \nu^{\prime}$, with the associated homotopies also respecting the decompositions and being fixed at $X$. Then we say the Poincaré embedding structures are equivalent to each other.

It is not difficult to see that the Poincaré embedding structure of 4.3 does not depend on the choice of the regular neighborhoods $U_{A}, U_{B}$, once the embeddings $(A, \partial A) \hookrightarrow\left(P, P_{0}\right)$ and $(B, \partial B) \hookrightarrow\left(Q, Q_{0}\right)$ are given, up to the equivalence between Poincaré embedding structures.

Definition 4.4. We will refer to the Poincaré embedding structure of 4.3, which is unique up to the equivalence, as the Poincaré embedding structure determined by the embeddings $(A, \partial A) \hookrightarrow\left(P, P_{0}\right)$ and $(B, \partial B) \hookrightarrow\left(Q, Q_{0}\right)$.

Now we summarize:

Proposition 4.5. If $\varphi$ is h-split along $\partial B$, then there is a unique Poincaré embedding structure on $\iota: Y \rightarrow(X, \partial X)$ determined by the embeddings $(A, \partial A) \hookrightarrow\left(P, P_{0}\right)$ and $(B, \partial B) \hookrightarrow\left(Q, Q_{0}\right)$.

We introduce another construction of Poincaré embedding structure on $\iota: Y \rightarrow$ $(X, \partial X)$ under a more general condition:

Construction 4.6. Assume there are maps: $H: P_{0} \times(I,\{0,1\}) \rightarrow Q_{0} \times(I,\{0,1\})$, and $F: \partial A \times(I,\{0,1\}) \rightarrow P_{0} \times(I,\{0,1\}), G: \partial B \times(I,\{0,1\}) \rightarrow Q_{0} \times(I,\{0,1\})$, which satisfy the following conditions:

i) $H_{0}$ is $h$-split along $\partial B$ and $H_{1}=\varphi$. Also $H(x, t)=(\varphi(x), t)$ for any $x \in \partial P_{0}$.

ii) $F_{0}$ is an embedding into Int $H_{0}^{-1} U_{\partial B} \subset P_{0}$, in which $U_{\partial B} \subset Q_{0}$ is an $h$ regular neighborhood of $\partial B$ associated to the $h$-splitting of $H_{0} . F_{1}$ is the inclusion $\iota_{\partial A}: \partial A \hookrightarrow P_{0}$.

iii) $H_{0} F_{0}=\varphi \iota_{\partial A}: \partial A \rightarrow Q_{0}$.

iv) Both $G_{0}$ and $G_{1}$ are the inclusion $\partial B \hookrightarrow Q_{0}$.

v) The following diagram commutes up to a homotopy rel $\partial A \times\{0,1\}$ :

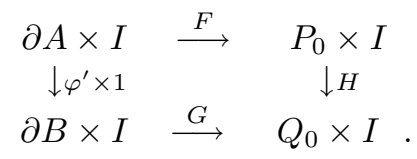

vi) Finally, $F, G$ are concordances. 
Note. $H_{s}: P_{0} \rightarrow Q_{0}, s=0,1$, denotes the map defined by $H(x, s)=\left(H_{s}(x), s\right)$. The maps $F_{s}, G_{s}, s=0,1$, should be understood likewise.

Under these conditions, we will define a Poincaré embedding structure on $\iota$ : $Y \rightarrow(X, \partial X)$ which is uniquely determined by $F, G$ and also by $H$. This is done as follows:

Choose collars of $\partial A \subset A$ and $\partial B \subset B$ respectively so that $A \cong \partial A \times I \cup_{A \times\{1\}} A^{\prime}$ and $B \cong \partial B \times I \cup_{B \times\{1\}} B^{\prime}$ for some submanifolds, $A^{\prime} \subset A, B^{\prime} \subset B$. Furthermore, we choose collars of $P_{0} \subset P$ and $Q_{0} \subset Q$ so that $P \cong P_{0} \times I \cup_{P_{0} \times\{1\}} P^{\prime}, Q \cong$ $Q_{0} \times I \cup_{Q_{0} \times\{1\}} Q^{\prime}$ and $A \cap P^{\prime}=A^{\prime}, B \cap Q^{\prime}=B^{\prime}$ and the inclusions, $A \hookrightarrow P$, $B \hookrightarrow Q$ restrict to $\partial A \times I \hookrightarrow P_{0} \times I, \partial B \times I \hookrightarrow Q_{0} \times I$ respectively so that they are just $\iota_{\partial A} \times 1, \iota_{\partial B} \times 1$, etc.

Let $\tilde{F}:(A, \partial A) \rightarrow\left(P, P_{0}\right)$ be given by $\tilde{F}(y)=y$ if $y \in A^{\prime}$ and $\tilde{F}(a, t)=F(a, t)$ if $(a, t) \in \partial A \times I$. Similarly, let $\tilde{G}:(B, \partial B) \rightarrow\left(Q, Q_{0}\right)$ be defined. Note that $\tilde{F}, \tilde{G}$ are well defined, and we may assume they are smooth embeddings.

Write $(\bar{X}, \partial \bar{X}) \equiv\left(P \cup_{H_{0}} Q, q\left(P_{1} \sqcup Q_{1}\right)\right)$. Note that $\partial \bar{X}=\partial X$. Then, the map $\iota_{1}: Y \rightarrow(\bar{X}, \partial X)$ is well defined by the rule: $\iota_{1}[y]=[\tilde{F}(y)]$ for any $y \in A$, $\iota_{1}[y]=[\tilde{G}(y)]$ for any $y \in B$ (note that $\iota_{\partial B} \varphi^{\prime}=H_{0} F_{0}$ by iii) above).

By 4.5 , there is a unique Poincaré embedding structure on $\iota_{1}: Y \rightarrow(\bar{X}, \partial \bar{X})$ determined by $\tilde{F}, \tilde{G}$.

The construction is complete except for the following:

Claim 4.A. There is a homotopy equivalence rel $\partial \bar{X}$,

$$
\alpha:(\bar{X}, \partial \bar{X}) \rightarrow(X, \partial X)
$$

so that $\alpha \iota_{1}$ is homotopic to $\iota$.

The proof exploits the condition v) in the above, and it is provided in Section 5 .

In fact, we can find maps $H, F, G$ satisfying all the conditions of 4.6, except for the requirement vi), simply by imposing a mild condition on the dimensions:

Lemma 4.7. Assume $\operatorname{dim} P-\operatorname{dim} A \geq 3$ and $2 \operatorname{dim} P \geq 3 \operatorname{dim} A+2$. Then, there are maps $H: P_{0} \times(I,\{0,1\}) \rightarrow Q_{0} \times(I,\{0,1\})$, and $F: \partial A \times(I,\{0,1\}) \rightarrow$ $P_{0} \times(I,\{0,1\}), G: \partial B \times(I,\{0,1\}) \rightarrow Q_{0} \times(I,\{0,1\})$, which satisfy the following conditions:

i) $H_{0}$ is h-split along $\partial B$ and $H_{1}=\varphi$. Also $H(x, t)=(\varphi(x), t)$ for any $x \in \partial P_{0}$.

ii) $F_{0}$ is an embedding into Int $H_{0}^{-1} U_{\partial B} \subset P_{0}$, in which $U_{\partial B} \subset Q_{0}$ is an $h$ regular neighborhood of $\partial B$ associated to the h-splitting of $H_{0} . F_{1}$ is the inclusion $\iota_{\partial A}: \partial A \hookrightarrow P_{0}$.

iii) $H_{0} F_{0}=\varphi \iota_{\partial A}: \partial A \rightarrow Q_{0}$.

iv) Both $G_{0}, G_{1}$ are the inclusion $\partial B \hookrightarrow Q_{0}$.

v) The following diagram commutes up to a homotopy rel $\partial A \times\{0,1\}$ :

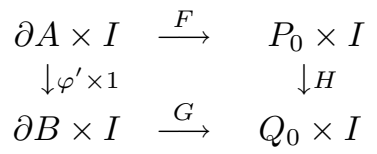

The proof of 4.7 is provided in the next section.

Note that $F$ and $G$ are homotopic to concordances rel $\partial A \times\{0,1\}$ and rel $\partial B \times$ $\{0,1\}$, respectively, if we assume $\operatorname{dim} P \geq 2 \operatorname{dim} A+1$ in addition to the dimensional conditions of 4.7. Therefore, we have 
Theorem 4.8. Let $\operatorname{dim} P \geq 2 \operatorname{dim} A+1$ and $\operatorname{dim} P \geq 6$. Then there is a Poincaré embedding structure on $\iota: Y \rightarrow(X, \partial X)$.

Now, by 1.5, we have

Proposition 4.9. Assume $\operatorname{dim} P=2 \operatorname{dim} A, \operatorname{dim} P-\operatorname{dim} A \geq 4$. Let $T \subset$ Int $Q_{0}$ be a codimension 1 compact submanifold without boundary with a trivial normal bundle. Assume $\varphi$ splits along T. Assume also $\partial A \subset \varphi^{-1} T$ and $\partial B \subset T$, and each path component of $T$ contains at most one path component of $\partial B$. Furthermore, assume

$$
\operatorname{Im}\left(\pi_{1}(\partial B) \rightarrow \pi_{1}(T)\right)<\pi_{1}(T) \text { satisfies the square root closed condition }
$$

at each path component. Then, $F, G$ of 4.7 can be chosen as concordances. In particular, there exists a Poincaré embedding structure on $\iota: Y \rightarrow(X, \partial X)$.

Note. In the above, 'splitting' is in the sense of Cappell ([2]). Say, let $Y$ be a connected closed smooth manifold, $X \subset Y$ a connected closed submanifold of codimension 1 and $Y_{0}, Y_{1}$, submanifolds of $Y$ such that $Y=Y_{0} \cup Y_{1}$ and $Y_{0} \cap Y_{1}=$ $X$. Then 'a homotopy equivalence $f: W \rightarrow Y$ from a closed manifold $W$ splits along $X$ ' means ' $f^{-1} X, f^{-1} Y_{i}, i=0,1$, are manifolds, and the restrictions of $f$ to $f^{-1} Y_{i} \rightarrow Y_{i}, i=0,1$, and to $f^{-1} X \rightarrow X$ are all homotopy equivalences.'

Proof of 4.9. Note that $\varphi^{-1} T \subset P_{0}$ and $T \subset Q_{0}$ respectively have neighborhoods diffeomorphic to $\varphi^{-1} T \times[-1,1]$ and $T \times[-1,1]$.

Apply Lemma 4.7 first to the inclusions

$$
\partial A \hookrightarrow \varphi^{-1} T, \quad \partial B \hookrightarrow T
$$

and the restriction of $\varphi$ to $\varphi^{-1} T \rightarrow T$. Thus, we may arrange that

$$
F: \partial A \times I \rightarrow P_{0}, \quad G: \partial B \times I \rightarrow Q_{0}
$$

factor respectively through the inclusions

$$
\varphi^{-1} T \hookrightarrow P_{0}, \quad T \hookrightarrow Q_{0} .
$$

However, the maps

$$
\bar{F}: \partial A \times I \rightarrow \varphi^{-1} T \times[-1,1] \times I, \quad \bar{G}: \partial B \times I \rightarrow T \times[-1,1] \times I
$$

respectively defined by

$$
\bar{F}(a, t)=(F(a, t), 0, t), \bar{G}(b, t)=(G(b, t), 0, t)
$$

are homotopic to an embedding respectively rel $\partial A \times\{0,1\}$ and rel $\partial B \times\{0,1\}$ by applying 1.5, which provides the concordances needed for us to apply 4.6.

This completes the proof of 4.9 .

Next we prove the main theorem, 1.3.

Proof of 1.3. For simplicity, we assume $\partial^{-} M_{0}=\partial^{+} M_{1}=\emptyset$. Then, note that we have $\partial^{+} M_{0}=\partial M_{0}$ and $\partial^{-} M_{1}=\partial M_{1}$, and also $X=M_{0} \cup_{f} M_{1}$.

For each $i=0,1, \partial M_{i}$ has a collar:

$$
M_{i} \cong M_{i}^{\prime} \cup_{\partial M_{i} \times\{1\}}\left(\partial M_{i} \times I\right)
$$

for some compact smooth manifold $M_{i}^{\prime} \subset M_{i}$. Therefore, presuming a fixed diffeomorphism, we shall identify $M_{i}$ with $M_{i}^{\prime} \cup\left(\partial M_{i} \times I\right)$. 


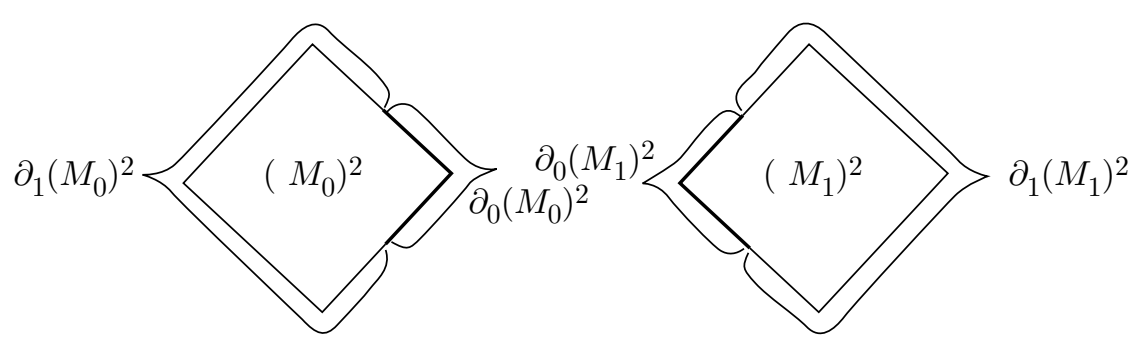

FiguRE 3

We shall denote $A \times A$ by $A^{2}$ for any space $A$. For example, $\left(M_{i}\right)^{2}=M_{i} \times M_{i}$, $\left(\partial M_{i}\right)^{2}=\partial M_{i} \times \partial M_{i}$, etc.. And let $\partial\left(M_{i}\right)^{2}$ denote the subspace

$$
\partial M_{i} \times M_{i} \cup M_{i} \times \partial M_{i}
$$

of $\left(M_{i}\right)^{2}$. We will also write

$$
\partial\left(M_{i}\right)^{2}=\left(\partial M_{i} \times\{0\}\right) \times M_{i} \cup M_{i} \times\left(\partial M_{i} \times\{0\}\right) .
$$

We define $\partial_{0}\left(M_{i}\right)^{2} \subset \partial\left(M_{i}\right)^{2}, i=0,1$, by

$$
\partial_{0}\left(M_{i}\right)^{2}=\left(\partial M_{i} \times\{0\}\right) \times\left(\partial M_{i} \times I\right) \cup\left(\partial M_{i} \times I\right) \times\left(\partial M_{i} \times\{0\}\right) .
$$

Also we define $\partial_{1}\left(M_{i}\right)^{2}$ as the closure of $\partial\left(M_{i}\right)^{2}-\partial_{0}\left(M_{i}\right)^{2}$ in $\partial\left(M_{i}\right)^{2}$. In other words, $\partial_{1}\left(M_{i}\right)^{2}$ is the space

$$
M_{i}^{\prime} \times\left(\partial M_{i} \times\{0\}\right) \cup\left(\partial M_{i} \times\{0\}\right) \times M_{i}^{\prime} .
$$

See Figure 3.

Now, consider the manifold triads $\left(\left(M_{i}\right)^{2} ; \partial_{0}\left(M_{i}\right)^{2}, \partial_{1}\left(M_{i}\right)^{2} ; \partial \partial_{1}\left(M_{i}\right)^{2}\right), i=0,1$, and the map

$$
\hat{f}:\left(\partial_{0}\left(M_{0}\right)^{2}, \partial \partial_{0}\left(M_{0}\right)^{2}\right) \longrightarrow\left(\partial_{0}\left(M_{1}\right)^{2}, \partial \partial_{0}\left(M_{1}\right)^{2}\right)
$$

defined by

$$
\hat{f}(x, s, y, t)=(f(y), t, f(x), s)
$$

for any $(x, s, y, t) \in\left(\partial M_{0} \times I\right)^{2}$ such that $s=0$ or $t=0$.

We introduce the following identification:

$$
\begin{aligned}
\partial_{0}\left(M_{i}\right)^{2}=\left(\partial M_{i} \times\{0\}\right) & \times\left(\partial M_{i} \times I\right) \cup\left(\partial M_{i} \times I\right) \times\left(\partial M_{i} \times\{0\}\right) \\
& \cong\left(\partial M_{i}\right)^{2} \times[-1,1]
\end{aligned}
$$

respectively given by the homeomorphisms

$$
\begin{array}{ll}
(x, s, y, t) \longrightarrow(x, y, s-t) & \text { if } i=0, \\
(x, s, y, t) \longrightarrow(y, x, t-s) & \text { if } i=1 .
\end{array}
$$

Then, up to these identifications, $\hat{f}$ as defined above is simply $f \times f \times 1$. Let $(N, \partial N)$ denote the pair

$$
\left(\left(M_{0}\right)^{2} \cup_{\hat{f}}\left(M_{1}\right)^{2}, \partial_{1}\left(M_{0}\right)^{2} \cup_{\hat{f}^{\prime}} \partial_{1}\left(M_{1}\right)^{2}\right),
$$

where $\hat{f}^{\prime}$ is the restriction of $\hat{f}$.

Note that there is a well-defined injection $\iota: X \rightarrow N$ given by the diagonal maps $\left(M_{i}, \partial M_{i}\right) \rightarrow\left(\left(M_{i}\right)^{2}, \partial_{0}\left(M_{i}\right)^{2}\right), i=0,1$. 


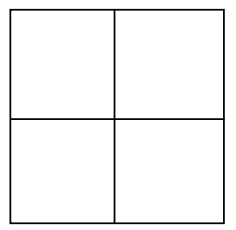

$X \times X$

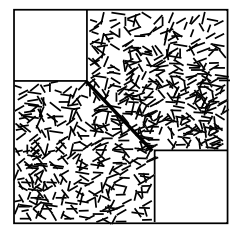

$\overline{X \times X}$

Figure 4

Claim 4.B. There is a space $\overline{X \times X}$ satisfying:

i) $\overline{X \times X}$ admits a decomposition $\overline{X \times X}=N \cup C$ in which the equality, $N \cap C=$ $\partial N$, holds and

ii) $(C, \partial N)$ is a Poincaré pair. Furthermore,

iii) there is a homotopy equivalence $h: \overline{X \times X} \rightarrow X \times X$ so that the diagram,

$$
\begin{array}{ccc}
X & \stackrel{\Delta}{ } & X \times X \\
\downarrow_{\iota} & & \uparrow h \\
N & \hookrightarrow & X \times X
\end{array}
$$

commutes, where $\Delta$ is the diagonal map (see Figure 4).

The proof of the claim is straightforward, and we provide only a sketch of it in the next section.

Then the assumptions of 4.9 is satisfied by $\iota: X=M_{0} \cup_{f} M_{1} \rightarrow\left(M_{0}\right)^{2} \cup_{\hat{f}}\left(M_{1}\right)^{2}$ if we set $T=\left(\partial M_{1}\right)^{2} \times\{0\}$. Thus, we may conclude there exists a Poincaré embedding structure on $\iota: X \rightarrow(N, \partial N)$, which is enough to prove Theorem 1.3.

Remark. The theorem is true in a more general setting. We could have replaced the manifold triads $\left(M_{i} ; \partial^{-} M_{i}, \partial^{+} M_{i} ; \emptyset\right)$ by the more general

$$
\left(M_{i} ; \partial^{-} M_{i}, \partial^{+} M_{i} ; \partial \partial^{-} M_{i}\right)
$$

and consider

$$
(X, Y) \simeq\left(M_{0} \cup_{f} M_{1}, q\left(\partial^{-} M_{0} \sqcup \partial^{+} M_{1}\right)\right),
$$

in which $f:\left(\partial^{+} M_{0}, \partial \partial^{+} M_{0}\right) \rightarrow\left(\partial^{-} M_{1}, \partial \partial^{-} M_{1}\right)$ is a homotopy equivalence and $q: M_{0} \sqcup M_{1} \rightarrow M_{0} \cup_{f} M_{1}$ is the quotient map.

\section{Proofs}

Proof of Claim 4.A. We construct $\alpha$ by applying 2.5. First of all, construct a homotopy equivalence,

$$
\alpha^{\prime}: P_{0} \times I \cup_{H_{0}} Q_{0} \rightarrow P_{0} \times I \cup_{H_{1}} Q_{0}
$$

rel $P_{0} \times\{1\} \cup q\left(\partial P_{0} \times I \sqcup Q_{0}\right)$ as in 2.5, assuming $\partial P_{0} \neq \emptyset$ (the simpler case $\partial P_{0}=\emptyset$ can be dealt with similarly). To be more specific, $P_{0}, Q_{0}$ here respectively correspond to $X, Y$ of 2.5. Furthermore, we use a decomposition of $P_{0}$ in such a way that both $A, B$ of 2.6 correspond to $\partial P_{0}$ and $C$, to $P_{0}-\partial P_{0} \times[0,1)$, where $\partial P_{0} \times[0,1)$ is identified with an open collar of $\partial P_{0} \subset P_{0}$.

Then we subsequently define $\alpha:(\bar{X}, \partial \bar{X}) \rightarrow(X, \partial X)$, so that $\alpha$ is the identity on $\bar{X}-\left(P_{0} \times[0,1) \cup_{H_{0}} Q_{0}\right)$ and $\alpha=\alpha^{\prime}$ on $P_{0} \times I \cup_{H_{0}} Q_{0}$.

Now we prove that $\alpha \iota_{1} \simeq \iota$. 
Since $F$ is an embedding and, by construction, $H F \simeq G\left(\varphi^{\prime} \times 1\right): \partial A \times I \rightarrow$ $Q_{0} \times I$ rel $\partial A \times\{0,1\}$, we may apply a cofibration argument to replace the map $H: P_{0} \times I \rightarrow Q_{0} \times I$ with a new one, up to homotopy rel $\partial P_{0} \times I \cup P_{0} \times\{0,1\}$, so that, still calling the new map $H$, we have

$$
H F=G\left(\varphi^{\prime} \times 1\right) \text {. }
$$

Thus we may assume $\alpha$ is constructed using such $H$. Then we have $\alpha \iota_{1} \equiv \iota$ on $A^{\prime} \sqcup B^{\prime} \subset Y$.

By construction, $\alpha$ restricts to

$$
\alpha^{\prime}: P_{0} \times I \cup_{H_{0}} Q_{0} \times I \longrightarrow P_{0} \times I \cup_{\varphi} Q_{0} \times I .
$$

Also $\iota_{1}$ restricts to

$$
\iota_{1}^{\prime}: \partial A \times I \cup_{\varphi^{\prime}} \partial B \times I \longrightarrow P_{0} \times I \cup_{H_{0}} Q_{0} \times I .
$$

On the other hand, we may assume the restriction of $\iota$ to

$$
\iota^{\prime}: \partial A \times I \cup_{\varphi^{\prime}} \partial B \times I \longrightarrow P_{0} \times I \cup_{\varphi} Q_{0} \times I
$$

is given by $\iota^{\prime}[y, t]=[y, t]$ for any $y \in \partial A \sqcup \partial B$. Now, to show $\alpha \iota_{1} \simeq \iota$, it is enough to prove that

$$
\alpha^{\prime} \iota_{1}^{\prime} \simeq \iota^{\prime} \operatorname{rel}(\partial A \sqcup \partial B) \times\{1\} .
$$

We may assume $p F(\partial A \times I) \subset P_{0}-\partial P_{0} \times[0,1)$, where $p: P_{0} \times I \rightarrow P_{0}$ is the projection. Then, by the definitions of $\alpha^{\prime}$ and $\iota_{1}^{\prime}$, it follows that

$$
\alpha^{\prime} \iota_{1}^{\prime}[y, t]=\left\{\begin{array}{cl}
{[y, 2 t-1]} & \text { if }(y, t) \in \partial A \times\left[\frac{1}{2}, 1\right], \\
{[H F(y, 2 t), 0]} & \text { if }(y, t) \in \partial A \times\left[0, \frac{1}{2}\right], \\
{[G(y, t)]} & \text { if }(y, t) \in \partial B \times I .
\end{array}\right.
$$

A homotopy

$$
\mathbf{H}:\left(\partial A \times I \cup_{\varphi^{\prime}} \partial B \times I\right) \times I \longrightarrow P_{0} \times I \cup_{\varphi} Q_{0} \times I
$$

from $\alpha^{\prime} \iota_{1}^{\prime}$ to $\iota_{1}^{\prime}$ rel $\left.(\partial A \sqcup \partial B) \times\{1\}\right)$ can be given, which 'cancels $F$ with $G$ ' as follows:

$\mathbf{H}$ is defined as follows: if $y \in \partial A$ and $t, u \in I$, then

$$
\mathbf{H}[y, t, u]=\left\{\begin{array}{cl}
[H F(y, 2 t+u), 0)] & \text { if } 0 \leq t \leq \frac{1-u}{2} \\
{\left[y, \frac{2 t-1+u}{1+u}\right]} & \text { if } \frac{1-u}{2} \leq t \leq 1,
\end{array}\right.
$$

and, if $y \in \partial B$ and $t, u \in I$, then

$$
\mathbf{H}[y, t, u]=\left\{\begin{array}{cc}
{[G(y, t+u), 0]} & \text { if } 0 \leq t \leq 1-u, \\
{[y, t]} & \text { if } 1-u \leq t \leq 1 .
\end{array}\right.
$$

Note that $\mathbf{H}$ is well-defined, in particular, since we have: $H F=G\left(\varphi^{\prime} \times 1\right)$.

We conclude that $\alpha \iota_{1}$ is homotopic to $\iota$.

To provide a proof of 4.7 , we shall use the following theorem in addition to 3.1.

Theorem 5.1 (S.E. Cappell). Let $Y$ be a Poincaré complex of dimension $n+1$, $n \geq 5$, and $X$ a closed connected sub-Poincaré complex of dimension $n$ of $Y$ for which there are connected subcomplexes $Y_{0}$ and $Y_{1}$ satisfying $Y=Y_{0} \cup Y_{1}$ and $X=$ $Y_{0} \cap Y_{1}$, and $\left(Y_{0}, X\right),\left(Y_{1}, X\right)$ are Poincaré pairs. Assume one of the homomorphisms $\pi_{1}(X) \rightarrow \pi_{1}\left(Y_{i}\right)$ is an isomorphism. Then any homotopy equivalence $f: W \rightarrow Y$, where $W$ is a smooth manifold, is splittable along $X$. 
In fact, 5.1 is a portion of what is stated in Theorem 1 of [2] and the footnote below it (also see [13]). Note that the square-root closed condition is not needed here.

Proof of Lemma 4.7. First, we construct the map $H: P_{0} \times I \rightarrow Q_{0} \times I$ so that it satisfies $i)$.

Let $\left(U_{B}, U_{\partial B}\right) \subset\left(Q, Q_{0}\right)$ be a regular neighborhood of $(B, \partial B)$ such that $U_{B} \cap$ $Q_{1}=\emptyset$. Then, since $\operatorname{dim} Q_{0}-\operatorname{dim} \partial B \geq 3$ by assumption, $\partial U_{\partial B} \hookrightarrow U_{\partial B}$ induces an isomorphism between fundamental groups. Assume each path component of $Q_{0}$ contains at most one path component of $B$. Then, by applying a relative version of 5.1, there is a homotopy $H^{\prime}: P_{0} \times I \rightarrow Q_{0}$ rel $\partial P_{0}$ such that $H_{0}^{\prime}$ splits along $\partial U_{\partial B}$ and $H_{1}=\varphi$. For the path components of $Q_{0}$ which contain more than one path component of $\partial B$, we apply the same theorem to the path components of $\partial B$, one by one, taking out the interior of the regular neighborhood from the target domain at each step. Then we define $H: P_{0} \times I \rightarrow Q_{0} \times I$ by the rule $H(x, t)=\left(H^{\prime}(x, t), t\right)$.

As the second step, we construct $F: \partial A \times I \rightarrow P_{0} \times I$ so that it satisfies $\left.i i\right)$.

Write $V_{\partial B} \equiv Q_{0}-$ Int $U_{\partial B}$ and set $U_{\partial A} \equiv H_{1}^{-1} U_{\partial B}, V_{\partial A} \equiv H_{1}^{-1} V_{\partial B}$. Let $\hat{H}_{0}$ be the inverse of $H_{0}:\left(P_{0}, \partial P_{0}\right) \rightarrow\left(Q_{0}, \partial Q_{0}\right)$, which preserves the splitting of $H_{0}$; that is, $\hat{H}_{0}$ restricts to $U_{\partial B} \rightarrow U_{\partial A}, V_{\partial B} \rightarrow V_{\partial A}, \partial U_{\partial B} \rightarrow \partial U_{\partial A}$ etc.

Let $\bar{e}$ be the composite

$$
\partial A \hookrightarrow\left(P_{0}, \partial P_{0}\right) \stackrel{H_{0}}{\longrightarrow}\left(Q_{0}, \partial Q_{0}\right) \stackrel{\hat{H}_{0}}{\longrightarrow}\left(P_{0}, \partial P_{0}\right) .
$$

Then it follows that $\bar{e}$ is homotopic to the inclusion $\iota_{\partial A}: \partial A \rightarrow P_{0}$, since $\hat{H}_{0} \varphi$ is homotopic to the identity.

Note that

$$
\bar{e} A \subset \operatorname{Int} U_{A} .
$$

The restriction $e$ of $\bar{e}$ to $A \rightarrow$ Int $U_{A}$ is a homotopy equivalence. Therefore, since $2 \operatorname{dim} P_{0} \geq 3(\operatorname{dim} A+1), e$ is homotopic to a smooth embedding by applying 3.1.

Thus, we have shown there is a homotopy $F^{\prime}: \partial A \times I \rightarrow P_{0}$ from $\bar{e}$ to $\iota_{\partial A}$. Define $F: \partial A \times I \rightarrow P_{0} \times I$ by the rule $F(x, t)=\left(F^{\prime}(x, t), t\right)$.

Now we modify $H: P_{0} \times I \rightarrow Q_{0} \times I$ so that $\left.i i i\right)$ can be satisfied.

Let $\iota_{\partial B}: \partial B \hookrightarrow Q_{0}, \iota:$ Int $U_{\partial B} \hookrightarrow Q_{0}$ denote the inclusions and $H_{0}^{\prime \prime}$ : Int $U_{\partial A} \rightarrow$ Int $U_{\partial B}$, the restriction of $H_{0}$. Note that $e: \partial A \rightarrow$ Int $U_{\partial A}$ is an embedding and $H_{0}^{\prime \prime} e: \partial A \rightarrow$ Int $U_{\partial B}$ is homotopic to $\iota_{\partial B} \varphi^{\prime}: \partial A \rightarrow \operatorname{Int} U_{\partial B}$. Therefore, by a cofibration argument, there is a homotopy from $H_{0}$ to a map, say $\tilde{H}_{0}$, so that $\tilde{H}_{0} e \equiv \varphi^{\prime}$ and $\tilde{H}_{0}$ preserves the splitting of $H_{0}$. We replace the homotopy $H^{\prime}$ by the new one, $H^{\prime}$, followed by the homotopy from $H_{0}$ to $\tilde{H}_{0}$. Keep calling it $H^{\prime}$. Then, we define $H: P_{0} \times I \rightarrow Q_{0} \times I$ by the rule $H(x, t)=\left(H^{\prime}(x, t), t\right)$.

Now we choose a $G: \partial B \times I \rightarrow Q_{0}$ so that $i v$ ) is satisfied.

Consider the base point preserving map,

$$
\left(\varphi^{\prime}\right)^{*}:\left(\operatorname{Map}\left(\partial B, Q_{0}\right), \iota_{\partial B}\right) \rightarrow\left(\operatorname{Map}\left(\partial A, Q_{0}\right), \iota_{\partial B} \varphi^{\prime}\right) .
$$

$\left(\varphi^{\prime}\right)^{*}$ is a homotopy equivalence since $\varphi^{\prime}$ is a homotopy equivalence. Note, in particular, that $\left(\varphi^{\prime}\right)^{*}$ induces an isomorphism between the fundamental groups. Therefore, there is a loop,

$$
g:(I,\{0,1\}) \rightarrow\left(\operatorname{Map}\left(\partial B, Q_{0}\right), \iota_{\partial B}\right)
$$


so that $\left(\varphi^{\prime}\right)^{*} g$ represents the loop ad $(H F)$ in the pointed space

$$
\left(\operatorname{Map}\left(\partial A, Q_{0}\right), \iota_{\partial B} \varphi^{\prime}\right) \text {, }
$$

where $\operatorname{ad}(H F)$ is defined by the rule $\operatorname{ad}(H F)(t)=H_{t} F_{t}$. Let $G^{\prime}: \partial B \times I \rightarrow Q_{0}$ be such that $G_{t}^{\prime}=g(t)$. Define $G: \partial B \times I \rightarrow Q_{0} \times I$ by the rule $G(x, t)=\left(G^{\prime}(x, t), t\right)$. This completes the proof of 4.7 .

Sketch of the proof of Claim 4.B. Write:

$$
K \equiv \partial M_{0} \times I \cup_{f} \partial M_{1} \times I .
$$

Since $K$ is a neighborhood of $\partial M_{1}=\partial M_{1} \times\{0\} \subset X, K \times K$ is a neighborhood of $\left(\partial M_{1}\right)^{2}$ in $X \times X$. We will write

$$
\begin{aligned}
\operatorname{Int} K & \equiv \partial M_{0} \times[0,1) \cup_{f} \partial M_{1} \times[0,1), \quad \text { Int } K^{2} \equiv(\operatorname{Int} K)^{2}, \\
\partial K & \equiv \partial M_{0} \times\{1\} \sqcup \partial M_{1} \times\{1\}, \\
\partial(K \times K) & \equiv\{([x, s],[y, t]) \in K \times K \mid s=1 \text { or } t=1\} .
\end{aligned}
$$

To construct $\overline{X \times X}$, we will replace $K \times K \subset X \times X$ by some other space, say $\overline{K \times K}$, which contains $\partial(K \times K)$ as a subspace and is homotopy equivalent to $K \times K$ rel $\partial(K \times K)$. In fact, we define $\overline{K \times K}$ as follows:

Note that $K \times K$ can be understood as a space formed by gluing the four spaces

$$
\begin{aligned}
& \left(\partial M_{0} \times I\right)^{2}, \quad\left(\partial M_{0} \times I\right) \times\left(\partial M_{1} \times I\right), \\
& \left(\partial M_{1} \times I\right) \times\left(\partial M_{0} \times I\right), \quad\left(\partial M_{1} \times I\right)^{2} .
\end{aligned}
$$

We define $\overline{K \times K}$, say, by rearranging the gluing (see the figure below Claim 4.B).

First we glue $\left(\partial M_{0} \times I\right) \times\left(\partial M_{1} \times I\right) \sqcup\left(\partial M_{1} \times I\right) \times\left(\partial M_{0} \times I\right)$ to $\left(\partial M_{1} \times I\right)^{2}$ using the gluing map:

$$
\begin{aligned}
h \equiv f \times & (1 \times \mu) \sqcup(1 \times \mu) \times f: \\
& \left(\partial M_{0} \times\{0\}\right) \times\left(\partial M_{1} \times I\right) \sqcup\left(\partial M_{1} \times I\right) \times\left(\partial M_{0} \times\{0\}\right) \\
& \longrightarrow\left(\partial M_{1} \times\{0\}\right) \times\left(\partial M_{1} \times I\right) \cup\left(\partial M_{1} \times I\right) \times\left(\partial M_{1} \times\{0\}\right),
\end{aligned}
$$

where 1 represents the identity maps and $\mu: I \rightarrow I$ is defined by $\mu(t)=\frac{1}{2}(t+1)$. Then we write $\widehat{K \times K}$ for the resulting space.

Consider the subspace $\partial_{0} \widehat{K \times K}$ of $\widehat{K \times K}$, defined as

$$
\begin{gathered}
\left(\left(\partial M_{1} \times\{0\}\right) \times\left(\partial M_{0} \times I\right)\right) \cup_{h^{\prime}}\left(\left(\partial M_{0} \times\left[0, \frac{1}{2}\right]\right) \times\left(\partial M_{1} \times\{0\}\right)\right) \\
\cup\left(\left(\partial M_{0} \times I\right) \times\left(\partial M_{1} \times\{0\}\right)\right) \cup_{h^{\prime \prime}}\left(\left(\partial M_{1} \times\{0\}\right) \times\left(\partial M_{0} \times\left[0, \frac{1}{2}\right]\right)\right),
\end{gathered}
$$

in which $h^{\prime}$ is the restriction of $(1 \times \mu) \times f$ to

$$
\left(\partial M_{1} \times\{0\}\right) \times\left(\partial M_{0} \times\{0\}\right) \longrightarrow\left(\partial M_{1} \times\left\{\frac{1}{2}\right\}\right) \times\left(\partial M_{1} \times\{0\}\right)
$$

and $h^{\prime \prime}$ is the restriction of $f \times(1 \times \mu)$ to

$$
\left(\partial M_{0} \times\{0\}\right) \times\left(\partial M_{1} \times\{0\}\right) \longrightarrow\left(\partial M_{1} \times\{0\}\right) \times\left(\partial M_{1} \times\left\{\frac{1}{2}\right\}\right) .
$$

Also consider the subspace $\partial_{0}\left(\partial M_{0} \times I\right)^{2}$ of $\left(\partial M_{0} \times I\right)^{2}$, which is defined as

$$
\left(\partial M_{0} \times\{0\}\right) \times\left(\partial M_{0} \times I\right) \cup\left(\partial M_{0} \times\{0\}\right) \times\left(\partial M_{0} \times I\right)
$$


Now we define the attaching map, $g: \partial_{0}\left(\partial M_{0} \times I\right)^{2} \longrightarrow \partial_{0} \widehat{K \times K}$, as follows:

$$
g(x, s, y, t)=\left\{\begin{aligned}
{[f(x), 0, y, 2 t-1] } & \text { if } s=0, \frac{1}{2} \leq t \leq 1 \\
{[f(y), t, f(x), 0] } & \text { if } s=0,0 \leq t \leq \frac{1}{2} \\
{[f(y), 0, f(x), s] } & \text { if } t=0,0 \leq s \leq \frac{1}{2} \\
{[x, 2 s-1, f(y), 0] } & \text { if } t=0, \frac{1}{2} \leq s \leq 1
\end{aligned}\right.
$$

To check the well-definedness of $g$ is straightforward.

Finally, we set

$$
\overline{K \times K} \equiv\left(\partial M_{0} \times I\right)^{2} \cup_{g} \widehat{K \times K} .
$$

Note that there is an obvious identification between

$$
\partial \overline{K \times K} \equiv\{[x, s, y, t] \in \overline{K \times K} \mid s=1 \text { or } t=1\}
$$

and

$$
\partial(K \times K)=\left\{[x, s, y, t] \in K^{2} \mid s=1 \text { or } t=1\right\} .
$$

Therefore, we may define $\overline{X \times X} \equiv(X \times X-\operatorname{Int}(K \times K)) \cup \overline{K \times K}$.

To show that $\overline{X \times X}$ satisfies $i), i i), i i i)$ of the claim is straightforward.

\section{REFERENCES}

1. R. Benlian et J. Wagoner, Type d'homotopie fibré et réduction structurale des fibrés vetoriels, C.R. Acad. Sci. Paris Sér. A-B 265 (1967), A 205 - A 209 MR 36:4576

2. S.E. Cappell, A splitting theorem for manifolds, Inventiones Math. 33 (1976), 69-17 MR 55:11274

3. A. Haefliger, Plongements différentiables de variétés dans variétés, Comment. Math. Helv. 36 (1961), 47-82 MR 26:3069

4. J.C. Hausmann and P. Vogel, Geometry on Poincaré spaces, Mathematical notes, Princeton University Press (1993) MR 95a:57042

5. J.P.E. Hodgson, The Whitney technique for Poincaré complex embeddings, Proc. AMS 35 (1972), 263-268 MR 46:2680

6. L. Jones, Patch spaces: a geometric representation for Poincaré spaces, Annals of Math. 97 (1973), 306-343; 102 (1975), 183-185. MR 47:4269, MR 52:11930

7. J.R. Klein, W. Richter and Shmuel Weinberger, A below middle dimension simply connected Poincaré Whitney embedding theorem, (to appear)

8. N. Levitt, Applications of Poincaré duality spaces to the topology of manifolds, Topology 11 (1972), 205-221 MR 45:4419

9. R.A. Piccinini, Lectures on homotopy theory, Mathematics Studies 171, North-Holland (1992) MR 93e:55001

10. V. Puppe, A remark on "homotopy fibrations", Manuscripta Math. 12 (1974), 113-120 MR 51:1808

11. E.H. Spanier, Algebraic topology, McGraw-Hill, New York (1966) MR 35:1007

12. C.T.C. Wall, Poincaré complexes I, Annals of Math. 86 (1967), 213-245 MR 36:880

13. Surgery on compact manifolds, Academic Press London \& New York (1970) MR 55: 4217

14. G.W. Whitehead, Elements of homotopy theory, Graduate Texts in Mathematics, SpringerVerlag, New York (1978) MR 80b:55001

15. H. Whitney, The singularities of a smooth $n$-manifold in $(2 n-1)$-space, Annals of Math. 45 (1944) 247-293 MR 5:274a

Department of Mathematics, Hanyang University, Seongdong-Gu, Seoul, Korea

E-mail address: yhbyun@fermat.hanyang.ac.kr 